\title{
La déconnexion régions-métropole au Québec : repenser les politiques publiques pour un développement territorial solidaire
}

\author{
Bruno Jean, professeur titulaire \\ Chaire de recherche du Canada en développement rural \\ Co-directeur du Centre de recherche sur le développement territorial (CRDT) \\ Université du Québec à Rimouski
}

\section{INTRODUCTION}

Le développement économique de tous les territoires du Québec ne peut reposer sur des stratégies de développement régional élaborées à la hâte pour répondre à des urgences du moment, qu'elles soient une réponse aux pressions populaires régionalistes ou aux politiques d'austérité des gouvernements. De telles interventions, souvent réactives et faiblement proactives, ne sont pas nécessairement mauvaises. En son temps, la stratégie de relance de l'économie montréalaise a permis une salutaire reconversion de cette économie métropolitaine, tout comme la stratégie de relance du développement des régionsressources a soutenu l'émergence du nouveau secteur des biotechnologies marines et celui de la valorisation de la tourbe dans le Bas-SaintLaurent. Le problème vient plutôt du fait qu'en concentrant les efforts et les ressources publiques dans un certain type de territoire, afin de compenser un handicap majeur comme la distance par exemple, une certaine forme de jalousie des acteurs économiques des autres territoires non visés ne tarde pas à se manifester. Tel semble être le cas avec la grogne, qui est revenue à la veille de l'annonce du budget du Québec, de certaines régions «centrales» face à la «stratégie des régionsressources », stratégie prétendument déloyale selon les dires des décideurs de ces régions.

Plus récemment, on a vu les positions de Raymond Bachand de l'Institut pour le développement du Québec reprenant le vieux modèle de développement du fameux rapport HMR postulant que le développement des régions passe par le développement de Montréal qui rediffuserait ce développement un peu partout $^{1}$ (voir aussi dans ce numéro le texte de A. Joyal). Et le Conseil du patronat en rajoute une couche en déclarant qu'il faut envisager de fermer certains territoires périphériques à l'occupation humaine au vu de leur prétendue non-viabilité économique. Nous pensons que le moment est venu de se donner les moyens d'élaborer une véritable politique proactive et prospective de soutien à un développement territorial solidaire.

Ce n'est pas parce que des notions comme la solidarité ou la démocratie sont reprises par des partis politiques qu'on ne peut plus les utiliser ou que, ce faisant, on manifesterait une adhésion au programme ce ces partis.

Nous avons proposé cette notion pour décrire une approche de développement régional reposant sur une saine compréhension de l'interdépendance régionale et sur la nécessaire solidarité interterritoriale pour remplacer la tendance quasi-naturelle à l'égoïsme territorial lors du colloque du CRDT à l'ACFAS en 2003. La solidarité dont il est question ici n'est pas une prise de position en faveur d'un parti politique, Québec solidaire, né bien après cette publication. Ce n'est pas parce que des notions comme la solidarité ou la démocratie sont reprises par des partis politiques qu'on ne peut plus les utiliser ou que, ce faisant, on manifesterait une adhésion au programme de ces partis $^{2}$.

Nous voulons esquisser ici les fondements d'une nouvelle approche pour l'élaboration et 
la mise en œuvre d'une véritable politique de développement territorial solidaire pour le Québec, une politique dont le Québec a besoin et qui reposera sur la reconnaissance de la nécessité de développer tous les territoires. Et comme ces territoires sont diversifiés sur le plan socioéconomique, ils réclament une politique avec des stratégies diversifiées, multiples et modulées en fonction d'un bon diagnostic territorial et d'une bonne identification de leur problématique de développement. Quant à la solidarité, elle ne devrait pas se réduire seulement à quelques jeux de péréquation dans l'allocation des ressources publiques inspirés par la compassion et la charité en faveur des territoires démunis considérés comme des espèces en voie de disparition. Cette politique devrait reposer sur une authentique solidarité alimentée par une meilleure compréhension des liens - de dépendance, d'indépendance ou d'interdépendance qui unissent les différents territoires du Québec, liens qui à l'évidence demeurent encore très mal connus.

\section{LA PROBLÉMATIQUE DES ÉCONOMIES RÉGIONALES AU QUÉBEC}

\section{Au fond, malgré un flottement dans les désignations, il y a un consensus large dans la communauté scientifique comme dans le monde des affaires pour affirmer que le Québec se découpe en ces trois grands types de régions.}

Le Québec ne se présente pas comme un assemblage de territoires homogènes dont les déterminants du développement économique régional seraient identiques. Si tel était le cas, une politique « mur à mur » de développement régional pourrait alors suffire à induire des effets positifs partout. Or, il y a une diversité de territoires et une diversité de connexions entre ces territoires, ce qui appelle à une diversité et une spécificité des approches en matière de politiques publiques. Un premier problème concerne la détermination des territoires et des catégories de territoires pour l'identification des problématiques de développement qui leur sont propres, les diagnostics territoriaux à réaliser, et finalement la politique de développement territorial approprié à construire pour chaque type de territoire.

Heureusement, les travaux des chercheurs des diverses sciences sociales ont produit un ensemble de connaissances sur les réalités territoriales du Québec qui ont permis d'établir que chacune des régions appartient à l'une ou l'autre de trois grandes entités territoriales, soit la région métropolitaine (Montréal et sa couronne urbaine), les régions centrales (ou intermédiaires) et les régions-ressources (ou périphériques). Au fond, malgré un flottement dans les désignations, il y a un consensus large dans la communauté scientifique comme dans le monde des affaires pour affirmer que le Québec se découpe en ces trois grands types de régions. Il nous semble plus approprié de parler de régions intermédiaires et de régions périphériques, plutôt que de régions centrales (comme la Coalition «SOS régions centrales » lancée à l'hiver 2006) et de régions-ressources, mais il s'agit là de conventions toujours révisables.

Une autre réalité à ne pas oublier, c'est la mouvance territoriale qui caractérise ce découpage régional qui bouge dans le temps; on constate ainsi un lent élargissement de la région métropolitaine intégrant des régions jusque-là considérées intermédiaires. On pourrait aussi soutenir que certains territoires considérés périphériques rejoignent les régions intermédiaires. Nous pensons ainsi à la partie ouest du BasSaint-Laurent, qui serait en train de s'intégrer dans les régions intermédiaires en se détachant de sa région périphérique d'appartenance, qu'on appelait l'Est-du-Québec à partir des années soixante pour revenir à la dualité BasSaint-Laurent Gaspésie, distinction symptomatique de cette évolution différentielle du Bas-Saint-Laurent. 


\section{UNE ÉCONOMIE QUÉBÉCOISE OUVERTE SUR LE MONDE}

La compétition qui affecte ces régions intermédiaires ne vient pas de ces régionsressources, elle vient d'autres régions du monde qui concurrencent sérieusement des pans entiers de leur secteur industriel et manufacturier.

Un des traits fondamentaux de l'économie québécoise, c'est son ouverture sur le monde. Et cela ne date pas de la période contemporaine de mondialisation des échanges économiques, mais bien des débuts de la société québécoise (avec la succession des staples qu'ont représentés successivement la traite des fourrures, puis le commerce $\mathrm{du}$ bois d'œuvre, du bois de sciage, du papier, l'énergie hydroélectrique, etc.). Cela signifie que la concurrence ne met pas tant les régions du Québec en compétition entre elles, comme dans une économie fermée, que dans une dynamique de compétition mondiale, une situation dans laquelle l'Asie s'impose actuellement et qui comporte son lot de phénomènes externes incontrôlables comme la variation des taux de change par exemple. Dans le débat récent sur la stratégie gouvernementale à l'égard des régionsressources, accuser celles-ci de concurrence déloyale à l'endroit des régions intermédiaires, c'est se tromper de cible. Car, sauf exception, la compétition qui affecte ces régions intermédiaires ne vient pas de ces régions-ressources, elle vient d'autres régions du monde qui concurrencent sérieusement des pans entiers de leur secteur industriel et manufacturier.

\section{LA DÉCONNEXION DE NOS RÉGIONS AVEC LA MÉTROPOLE}

Une caractéristique majeure de la structuration économique du Québec peut se résumer par ce que l'on peut appeler la déconnexion entre l'économie de la métropole et celles des régions. Les modèles théoriques de diffusion spatiale de l'activité économique, depuis les travaux pionniers des allemands Von Thunen, Christaller et Losch, ont bien établi le lien symbiotique entre une métropole et sa périphérie et qui fait en sorte que les deux territoires, mêmes dans une relation de dépendance, se confortent mutuellement dans leur dynamique propre de développement. Or, les secteurs porteurs de la nouvelle économie montréalaise comme la pharmaceutique, l'aéronautique, le génie ou encore le multimédia, ne diffusent pas (par de la sous-traitance qui crée des emplois) dans les régions périphériques.

\section{De nombreuses théories ou doctrines en développement régional misent pourtant sur la consolidation des pôles de croissance en postulant qu'il y aura diffusion de l'activité économique à partir de ces centres.}

À l'inverse, l'économie des ressources naturelles de ces territoires ne génère que peu de retombées dans l'économie métropolitaine. Nous sommes cons- cients qu'une telle affirmation n'est pas complètement vraie, car que serait cette économie sans toutes les ressources en provenance des régions qui assurent la prospérité de la métropole. Ce que nous voulons dire ici, c'est que les retombées y sont bien plus faibles qu'on pourrait s'attendre à cause cette déconnexion relative. En effet, de nombreuses théories ou doctrines en développement régional misent pourtant sur la consolidation des pôles de croissance en postulant qu'il y aura diffusion de l'activité économique à partir de ces centres. En 1970, le fameux rapport Higgins-Martin-Renaud avait fait grand bruit dans les régions périphériques en proposant de consolider les investissements publics en matière de développement économique régional sur la métropole montréalaise; il s'agissait d'une approche ayant complètement éludé la prise en compte de cette réalité fondamentale, mais mal comprise de la déconnexion entre l'économie métropolitaine et l'économie des régions périphériques. En conséquence, les effets anticipés pour les régions périphériques ne se sont jamais concrétisés. Ce seul exemple illustre bien l'insuffisance d'une politique de développement territorial qui serait unique et uniforme, car les territoires du Québec, désarticulés entre eux, le sont davantage avec l'économie et les marchés continentaux ou mondiaux que dans le cadre d'un espace économique autonome québécois. 


\section{LES CONSÉQUENCES DES POLITIQUES SECTORIELLES}

\section{Il faut maintenant passer d'une approche d'assistance à une approche basée sur une solidarité bien comprise, soit la solidarité qui naît de la compréhension des liens d'interdépendance entre les territoires du Québec.}

Enfin, une autre dimension de la problématique du développement régional au Québec réside dans une autre forme de déconnexion, celle entre les politiques économiques sectorielles et la politique régionale. Encensés par un discours politique parfois tonitruant, force est d'admettre que bien des efforts en matière de développement des régions ont souvent des effets ou des impacts fort modestes en comparaison avec certaines décisions et politiques économiques sectorielles qui elles cependant peuvent avoir des effets régionaux considérables, d'autant par exemple que les différents secteurs industriels ont tendance à se concentrer spatialement dans des espaces spécialisés. C'est ainsi que pendant longtemps, on a pensé régler les problèmes des régions rurales avec des politiques agricoles. Aujourd'hui, soutenir résolument le secteur de la pharmaceutique et de l'aéronautique, cela revient sans le dire à soutenir le développement de la région métropolitaine tout comme l'aide au secteur minier conforte au fond l'AbitibiTémiscamingue et la Côte-Nord. Il faut donc mieux prendre en compte la dimension territoriale des politiques économiques. Maintenir cette déconnexion entre la politique économique sectorielle et la politique régionale, cela revient à traiter l'ensemble de la question du développement régional par des politiques d'assistance à des régions en difficultés et pour lesquelles on ne pense pas qu'il soit possible d'initier et d'accompagner un véritable processus de développement territorial. Il faut maintenant passer d'une approche d'assistance à une approche basée sur une solidarité bien comprise, soit la solidarité qui naît de la compréhension des liens d'interdépendance entre les territoires du Québec.

\section{QUELQUES ÉL.ÉMENTS D'UN DIAGNOSTIC TERRITORIAL POUR LES TROIS TYPES DE RÉGIONS}

\section{La nécessité de politiques publiques axées vers une saine gestion de ces ressources (et d'abord leur reconstitution) et la mise en œuvre d'approches relevant du développement durable.}

Les régions périphériques, cela est bien connu et documenté, subissent de nombreux handicaps liés à la structure économique (dépendance au secteur primaire), à l'éloignement des marchés et bientôt à la pénurie de main-d'œuvre qui frappera tout le Québec. Là où c'est possible, une plus grande transformation des matières premières doit être envisagée de même qu'une plus grande valorisation des sous-produits tant de l'industrie forestière que de celle de la pêche. La compensation de ces handicaps tout comme le soutien à la constitution d'un secteur industriel dynamique participant à la nouvelle économie justifie des politiques publiques comme l'actuelle stratégie de développement des régionsressources. Mais l'on constate aussi que les ressources s'épuisent ou disparaissent dans les régions en question, comme le signale A. Joyal avec l'exemple de la Gaspésie, au point où leur désignation risque de perdre son sens. D'où la nécessité de politiques publiques axées vers une saine gestion de ces ressources (et d'abord leur reconstitution) et la mise en œuvre d'approches relevant du développement durable.

Un certain nombre d'économistes rappellent le rôle et l'importance persistante, voire la pertinence, d'une « économie matérielle » face à la supposée généralisation et l'hégémonie d'une économie du savoir plus immatérielle. On aura encore besoin de bois pour construire des bâtiments et des meubles, de produits agricoles pour se nourrir, etc. Ce qui donne à penser que les régions périphériques pourraient redevenir prospères dans le cadre d'une telle économie qui 
valoriserait à leur juste valeur les ressources naturelles contrairement à ce qu'écrit dans ces pages mon collègue A. Joyal qui ne semble miser que sur la diversification économique. Il faut également rappeler la pertinence d'une approche qui fonde le développement local sur l'identification et la mise en valeur des ressources « immobiles » d'un territoire, ressources qui sont souvent fort mal connues.

\section{QUELLE STRATÉGIE POUR LES RÉGIONS INTERMÉDIAIRES?}

\section{Une telle démarche qui ravive les égoïsmes territoriaux est tout le contraire d'une approche de développement territorial solidaire.}

Par ailleurs, les régions intermédiaires ou dites « centrales » vivent actuellement une période de transition fort difficile, car ces territoires, qui sont souvent les hôtes de secteurs vulnérables de l'économie manufacturière, sont directement aux prises avec certaines conséquences de la mondialisation. La reconversion économique radicale depuis quelques décennies d'une ville comme Drummondville, ville jadis forte sur le plan de l'industrie textile, donne à penser qu'il a y des raisons valables d'espérer. Cette municipalité illustre à quel point l'adaptation à la nouvelle économie mondialisée est possible avec la constitution de PME de «classe mondiale». Or, dans plusieurs régions intermédiaires, ce ne sont pas tous les entrepreneurs qui s'inscrivent dans un processus d'adaptation à la nouvelle réalité économique. Certains perçoivent de la concurrence provenant de leurs voisins immédiats alors que cette concurrence vient aussi et peut-être davantage des quatre coins de la planète. Dans le contexte de ces régions intermédiaires qui n'ont pratiquement jamais bénéficié du soutien étatique au développement régional dans le passé, en étant souvent la composante oubliée des stratégies visant ou bien la métropole ou bien les régions périphériques, une politique de développement régional doit être une politique de soutien à l'adaptation, à l'innovation et à la reconversion industrielles. Quand ces mêmes régions réclament l'abolition des mesures fiscales favorisant les entreprises dans les régions périphériques, elles dénoncent d'une main ce qu'elles réclament de l'autre, soit la modulation des programmes en faveur des diverses réalités régionales au Québec. Une telle démarche qui ravive les égoïsmes territoriaux est tout le contraire d'une approche de développement territorial solidaire.

\section{DE NOUVELLES POSSIBILITÉS POUR LA MÉTROPOLE}

\section{L'économie de la métropole a connu une \\ heureuse reconversion sur un ensemble de secteurs-clés extrêmement dynamiques, même si ces secteurs diffusent très peu dans les régions périphériques.}

Finalement, suite aux nombreux efforts des acteurs concernés et du gouvernement du Québec, l'économie de la métropole a connu une heureuse reconversion sur un ensemble de secteurs-clés extrêmement dynamiques, même si ces secteurs diffusent très peu dans les régions périphériques. Et s'il a été question plus haut de cette déconnexion entre l'économie de la métropole et celles des régions, il importe également de relever les exceptions qui confirment la règle : il ne faut pas oublier que près de la moitié des emplois et de la valeur ajoutée du secteur agroalimentaire québécois se concentrent dans la grande région de Montréal. Dans l'arrondissement Mercier/Hochelaga-Maisonneuve, c'està-dire au plein cour de Montréal, le CIDIM (Conseil des industries bioalimentaires de l'île de Montréal) a déjà annoncé l'intention de créer un «pôle bioalimentaire » de 1500 emplois. Avec le phénomène général de la métropolisation des économies, soutenir le développement de notre métropole demeure donc un impératif. Par ailleurs, il faudra aussi chercher des voies par lesquelles cette économie pourrait profiter davantage aux autres régions tout autant que ces dernières pourraient susciter des retombées plus importantes pour la métropole. 


\section{CONCLUSION: LE CADRE DE RÉFÉRENCE D'UNE POLITIQUE DE DÉVELOPPEMENT TERRITORIAL SOLIDAIRE}

\section{L'équité me semble un principe plus pertinent que la traditionnelle lutte aux disparités régionales, lutte perdue d'avance.}

Une politique publique de développement régional, comme on a pu le constater trop souvent dans le passé, pose la question des niveaux de juridictions où elle doit se définir. Le développement régional est-il un objet de juridiction fédérale ou provinciale? Avec la revendication historique pour la décentralisation portée par les défuntes Conférences régionales des élus, on aurait pu assister à l'émergence d'un nouveau palier de juridiction, celui de niveau régional comme tel? Sans entrer au cour de ces débats dont une partie concerne le rôle des organismes publics comme leviers de développement régional (on pense ainsi aux impacts régionaux décisifs des sociétés d'État comme HydroQuébec, Rexfor, Soquia et surtout la Caisse et dépôts et de la placement), il s'agira de penser ici à un cadre de référence qui met l'accent sur la nécessité d'une triple stratégie d'action en fonction de nos trois grands types de territoires et qui repose sur un certain nombre de principes devant guider les modalités particulières des interventions mises en œuvre.

Un des premiers principes est certainement celui de l'équité, ou autrement dit, le principe de solidarité, entre les territoires du Québec, qui postule implicitement la reconnaissance de la nécessité d'une occupation dynamique de ces territoires (en conformité avec la politique nationale de la ruralité malheureusement affaiblie sinon vidée de sa substance avec l'austérité actuelle). L'équité me semble un principe plus pertinent que la traditionnelle lutte aux disparités régionales, lutte perdue d'avance. Car ce n'est pas la réduction des disparités qu'il faut viser, mais une saine mise en valeur de ces différences régionales, base de leur développement, en misant entre autres sur «les ressources immobiles » de ces territoires. Un second principe, c'est le développement durable et on pourrait donc parler ici d'une politique de développement territorial durable des régions du Québec. Il faut aussi mentionner ici le principe de subsidiarité et l'imputabilité, mais il faut parler aussi de citoyenneté, un concept mal compris. Car les populations des régions devraient davantage, comme certaines minorités visibles, revendiquer le respect par les pouvoirs publics des droits que leur ouvre la citoyenneté; autrement dit, ils ne peuvent pas être traités en citoyens de seconde zone. Par ailleurs, on s'étonne que nos gouvernements, qui ont d'emblée un mandat de maintien de la cohésion sociale, ne légitiment pas l'utilisation des ressources publiques pour le développement régional au nom de ce grand principe qui est au cœur des finalités de l'action publique, et d'une action publique qui devrait être davantage territorialisée; en disant cela, nous évoquons ici un dernier principe d'une telle politique de développement territorial solidaire.

\section{BIBLIOGRAPHIE ET NOTES}

\footnotetext{
${ }^{1}$ Voir notre réaction à cette proposition dans Le Devoir : Bruno JEAN et Marie-Joelle BRASSARD, Il faut nuancer la thèse de Montréal, locomotive, Section «La réplique » de la page Idées, Montréal, Le Devoir, 18 décembre 2014, p. A-7

${ }^{2}$ Des éléments de la communication ont été repris dans un article dans Le Devoir. Voir : Bruno JEAN, « Le développement territorial : de l'égoïsme.
} 